\title{
DETERMINAÇÃO DA DIFUSIVIDADE EFETIVA E DA ENERGIA DE ATIVAÇÃO DE SEMENTES DE UVA BORDÔ
}

\author{
M. P. SILVA ${ }^{1}$, A. T. SALVADOR ${ }^{1}$, J. G. SGORLON ${ }^{1}$, M. C. S.GOMES ${ }^{1}$ e M. L. \\ MENEZES $^{1}$ \\ ${ }^{1}$ Universidade Tecnológica Federal do Paraná, Departamento de Engenharia Química \\ E-mail para contato: marcoss@ alunos.utfpr.edu.br
}

\begin{abstract}
RESUMO - É substancial a obtenção de informações teóricas a respeito do comportamento de cada produto durante o processo de secagem para o seu desenvolvimento e otimização. Sendo assim, o objetivo deste trabalho foi determinar e avaliar a difusividade efetiva e a energia de ativação do processo de secagem de sementes de uva Bordô, analisando a influência da temperatura no processo. Foi utilizado o modelo da difusão com a $2^{\mathrm{a}}$ Lei de Fick para descrever os dados de secagem e determinar o coeficiente de difusividade das sementes, além disso, a energia de ativação da difusão líquida durante o processo foi determinada utilizando-se a equação de Arrhenius. Verificou-se que a difusividade efetiva aumenta com o acréscimo da temperatura do ar de secagem, apresentando magnitudes entre $3,66 \times 10^{-11}$ e $2,88 \times 10^{-10} \mathrm{~m}^{2} \mathrm{~s}^{-1}$, para o intervalo de temperatura de 40 a $80{ }^{\circ} \mathrm{C}$, respectivamente. Valores esses que estão de acordo com o previsto na literatura para alimentos, entre $10^{-9}$ e $10^{-11}$. A energia de ativação apresentou valor de $42,1421 \mathrm{~kJ} \mathrm{~mol}^{-1}$, indicando que processo de difusão é basicamente lento.
\end{abstract}

\section{INTRODUÇÃO}

A viticultura brasileira surgiu em meio à colonização portuguesa, tornando-se uma atividade comercial no século XX. Desde então, houve uma grande ampliação da fronteira vitícola e a uva tornou-se uma alternativa econômica promissora. Atualmente, no país há uma grande variabilidade de castas, são mais de 120 cultivares de Vítis Vinífera e mais de 40 de variedades de uva americanas (Camargo; Tonietto; Hoffmann, 2011).

A semente da uva corresponde em torno de $5 \%$ do peso da fruta, assim, em média, anualmente são geradas mais de setenta e cinco mil toneladas desse resíduo só em território brasileiro (Choi e Lee, 2009). Além disso, Costa e Belchior (1972) descrevem que dos subprodutos resultantes da produção de $100 \mathrm{~L}$ de vinho, $4 \mathrm{~kg}$ correspondem às grainhas (ou sementes).

Objetivando-se encontrar soluções viáveis para o aproveitamento desse resíduo, a produção do óleo de sementes de uva cultivadas no país apresenta-se como uma alternativa promissora, uma vez que as sementes apresentam teores de óleos que oscilam de 13 a $20 \%$ dependendo da variedade, e este óleo é rico em ácidos graxos insaturados e antioxidantes (Fernandes et al., 2013). 
A secagem das sementes previamente ao processo de extração do óleo é essencial para diminuir a atividade da água na matéria-prima, estabilizando-a e consequentemente, retardando a degradação química e microbiana, prolongando assim, a sua vida útil (Roberts, Kidd e PadillaZakour, 2008).

A secagem de sólidos é uma das mais antigas e usuais operações unitárias, sendo caracterizada como um processo de retirada de água por evaporação no qual o equilíbrio entre a temperatura, a circulação e umidade relativa do ar define o processo (McCABE et al., 1976). A difusão de água durante a secagem é um processo complexo que pode envolver diferentes mecanismos, como a difusão capilar, molecular, de superfície, térmica e fluxo hidrodinâmico. Assim, pelo fato dessa não ser intrínseca ao produto, variando de acordo com as condições, é nomeada de difusividade efetiva (Goneli et al., 2009).

Diante do destaque da cultura da uva no cenário nacional e internacional, aliado à possibilidade de extração do óleo de sua semente, um resíduo da indústria vinícola, e a necessidade da etapa de secagem prévio à este processo, o objetivo geral deste trabalho é determinar e avaliar a difusividade efetiva e a energia de ativação do processo de secagem de sementes de uva Bordô, analisando a influência da temperatura no processo, uma vez que torna-se imprescindível a obtenção de informações teóricas a respeito do comportamento de cada produto durante a remoção da água para o desenvolvimento e aperfeiçoamento de equipamentos utilizados para a secagem de grãos e sementes.

\section{METODOLOGIA}

\subsection{Materiais}

As sementes de uva da variedade Bordô foram adquiridas na Vinícola Vinhos Randon Ltda, localizada na cidade de Pinheiro Preto/SC. O período de safra foi de meados de dezembro de 2010 até final de março de 2011.

\subsection{Métodos}

As sementes foram limpas e secas à temperatura ambiente, embaladas em sacos plásticos e armazenadas a $-15^{\circ} \mathrm{C}$. As sementes de uva foram submetidas à secagem em uma estufa (Solab, SL-102), sendo analisada a influência da temperatura no comportamento das curvas de secagem. As amostras de semente de uva foram secas, em triplicata, nas temperaturas de $40,50,60,70$ e $80{ }^{\circ} \mathrm{C}$. Na temperatura de secagem desejada e a cada 1 minuto, foi realizada a pesagem da amostra em balança analítica. A partir de 30 minutos de cada ensaio de secagem, o intervalo de tempo de pesagem passou a ser a cada 2 minutos. Este processo foi realizado até que três pesagens consecutivas fornecessem valores de massa constantes. A umidade em cada instante foi determinada pela Equação 1.

$$
X(b . s)=\left(M(t)-M_{\text {seca }}\right) / M_{\text {seca }}
$$

Em que: X(b.s) é a umidade em cada instante, (b.s); M(t) é a massa da amostra em cada tempo, (g) e Mseca é a massa seca das sementes obtida em estufa, (g). 
Foi utilizado o modelo da difusão com a $2^{\text {a }}$ Lei de Fick para descrever os dados de secagem e determinar a difusividade efetiva das sementes. A $2^{\text {a }}$ lei de Fick define uma difusividade molecular e não uma difusividade efetiva. Esta última é usada para levar em consideração os diversos mecanismos de transferência de umidade presentes em meios porosos e que não são previstos na equação original, conforme apresentado na Equação 2.

$$
\frac{\partial X}{\partial t}=D_{\text {eff }} \frac{\partial^{2} X}{\partial z^{2}}
$$

Em que: $\mathrm{X}$ é a fração mássica ou molar de um constituinte; t é o tempo e $\mathrm{D}_{\text {eff }}$ a difusividade efetiva.

Desprezando-se a interdifusão, uma vez que a água migra dentro de uma matriz fixa e assumindo a resistência externa desprezível, tem-se as seguintes condições inicial e de contorno:

- Distribuição inicial de umidade uniforme: $\mathrm{t}=0,0 \leq \mathrm{z} \leq$ espessura (L), $\mathrm{X}=\mathrm{X}_{0}$;

- Condição de contorno na base do sólido: $\mathrm{z}=0, \mathrm{t} \geq 0, \frac{\partial X}{\partial z}=0$;

- Condição de contorno na superfície do sólido: $\mathrm{z}=\mathrm{L}, \mathrm{t} \geq 0$ : $\mathrm{X}_{\mathrm{L}}=\mathrm{X}_{\mathrm{e}}$.

A solução analítica integrada ao longo da distância resulta na Equação 3 com a qual é possível determinar a difusividade efetiva, a qual é válida para meio poroso com difusividade constante (Crank, 1975).

$$
\frac{\mathrm{X}-\mathrm{Xe}}{\mathrm{Xo}-\mathrm{Xe}}=\frac{8}{\pi^{2}} \sum_{\mathrm{n}=1}^{\infty} \frac{1}{(2 \mathrm{n}-1)^{2}} \exp \left(-\frac{(2 \mathrm{n}-1)^{2} \pi^{2} \mathrm{t} \mathrm{D}_{\mathrm{eff}}}{4 \mathrm{~L}^{2}}\right)
$$

Em que: $\mathrm{X}, \mathrm{X}_{0}$ e $\mathrm{X}_{\mathrm{e}}$ representam, respectivamente, o teor de água no tempo $\mathrm{t}$, inicial e no equilíbrio em $\mathrm{kg} \mathrm{H}_{2} \mathrm{O} / \mathrm{kg} \mathrm{m}_{\text {seca }}$; $\mathrm{D}_{\text {eff }}$ é o coeficiente de difusividade, m/s; t é o tempo em s; Lé a semi-espessura da amostra, em $\mathrm{m}$.

Para tempos longos de secagem, considera-se $n=1$ e a Equação 3 pode ser reduzida a apenas ao primeiro termo da série, obtendo-se a Equação 4.

$$
\frac{\mathrm{X}-\mathrm{Xe}}{\mathrm{X} 0-\mathrm{Xe}}=\frac{8}{\pi^{2}}\left(-\frac{\pi^{2} \mathrm{tD} \text { eff }}{4 \mathrm{~L}^{2}}\right)
$$

Linearizando a Equação 4, e plotando-se o gráfico de ln $\left(\mathrm{X}-\mathrm{Xe} / \mathrm{X}_{0}\right.$ - Xe) em função do tempo, obtém-se a inclinação (K0) de acordo com a Equação 5, a partir da qual foi possível se determinar a difusividade efetiva (Deff ) que engloba os efeitos de todos os fenômenos que podem intervir sobre a migração da água, sendo dependente da temperatura para cada condição de secagem.

$$
\mathrm{K}_{0}=\frac{\pi^{2} \mathrm{D}_{\text {eff }}}{4 \mathrm{~L}^{2}}
$$

Por fim, a Equação 6 foi utilizada para cálculo da energia de ativação para cada condição de secagem. 


$$
\mathrm{D}_{\text {eff }}=\mathrm{D}_{0} \exp \left(\frac{-\mathrm{Ea}}{\mathrm{RT}}\right)
$$

Em que: Do é o fator pré-exponencial da equação de Arrhenius ( $\left.\mathrm{m}^{2} / \mathrm{s}\right) ; \mathrm{R}$, a constante universal dos gases $(8,314 \mathrm{~J} / \mathrm{mol} \mathrm{K})$; T, a temperatura absoluta $(\mathrm{K})$ e Ea, a energia de ativação $(\mathrm{kJ} / \mathrm{mol})$.

\section{RESULTADOS E DISCUSSÕES}

Usando a Equação 3 proposta por Crank (1975), a qual é a solução analítica da $2^{a}$ Lei de Fick, uma equação constitutiva empírica que relaciona a taxa de difusão molecular com o gradiente de concentração, e em posse da espessura da amostra de $3,02 \mathrm{~mm}$ a qual foi medida

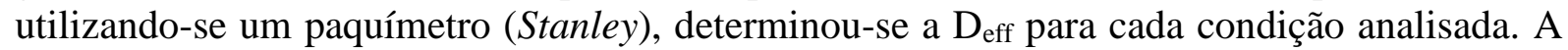
Tabela 1 apresenta, respectivamente, os valores de $\mathrm{K}_{0}$ e da difusividade efetiva para cada temperatura de secagem.

Tabela 1 - $\mathrm{K}_{0}$ e $\mathrm{D}_{\text {eff }}$ para cada temperatura de secagem

\begin{tabular}{c|c|c}
\hline Temperatura $(\mathrm{K})$ & $\mathrm{K}_{0}\left(10^{-4} \mathrm{~s}^{-1}\right)$ & $\mathrm{D}_{\text {eff }}\left(10^{-10} \mathrm{~m}^{2} \mathrm{~s}^{-1}\right)$ \\
\hline 313,15 & $-0,40$ & 0,37 \\
\hline 323,15 & $-1,58$ & 1,46 \\
\hline 333,15 & $-1,74$ & 1,61 \\
\hline 343,15 & $-2,36$ & 2,18 \\
\hline 353,15 & $-3,12$ & 2,88 \\
\hline
\end{tabular}

Por meio da Figura 1, pode-se observar um comportamento praticamente linear $\left(\mathrm{R}^{2}=\right.$ 0,9515) da difusividade em relação à temperatura.

Figura 1 - Efeito da temperatura na difusividade efetiva

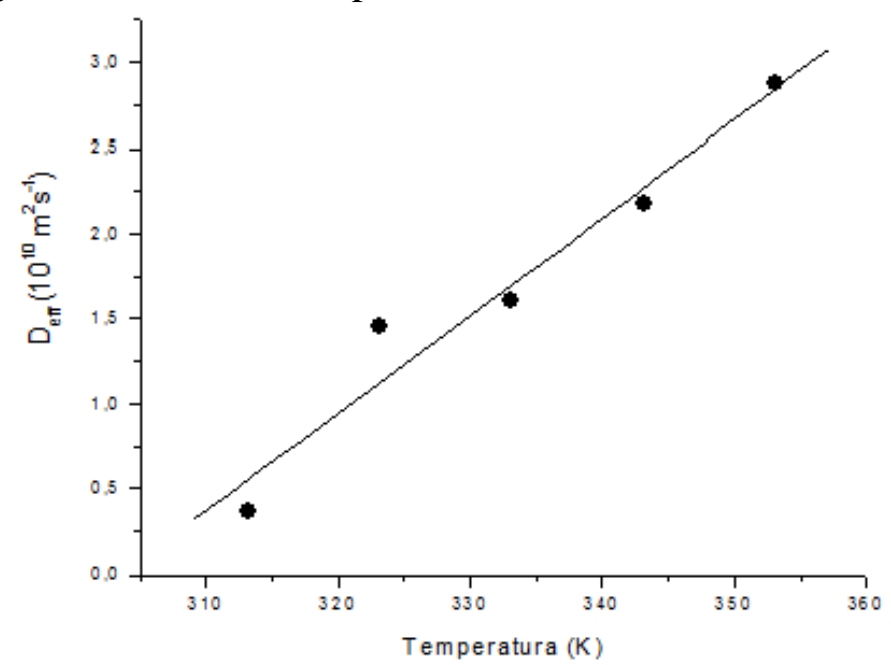

De acordo com os resultados apresentados na Tabela 1 e na Figura 1, pode-se verificar que com o aumento da temperatura ocorre, também, o aumento da difusividade efetiva. Esta relação pode ser explicada tendo em vista que a $D_{\text {eff }}$ representa a velocidade com que a água migra do interior para a superfície do material, sendo então vaporizada. O aumento da $D_{\text {eff }}$ com a temperatura é um resultado clássico e é explicado pelo fato da migração de umidade 
envolver mecanismos de difusão da água líquida e de vapor que são favorecidos pelo aumento da temperatura.

Além disso, observa-se que as difusividades efetivas apresentaram valores da ordem de grandeza de $10^{-10}$ e $10^{-11}$, o que está de acordo com o previsto na literatura para alimentos, entre $10^{-9}$ e $10^{-11}$ (Madamba et al., 1996).

A energia de ativação foi determinada linearizando-se a Equação 6, e plotando-se o gráfico de ln $\left(\mathrm{D}_{\text {eff }}\right)$ em função do inverso da temperatura $(1 / \mathrm{T})$, apresentado na Figura 2, no qual o coeficiente angular da reta fornece a razão entre a energia de ativação e a constante dos gases ideais.

Figura 2 - Determinação da Energia de Ativação

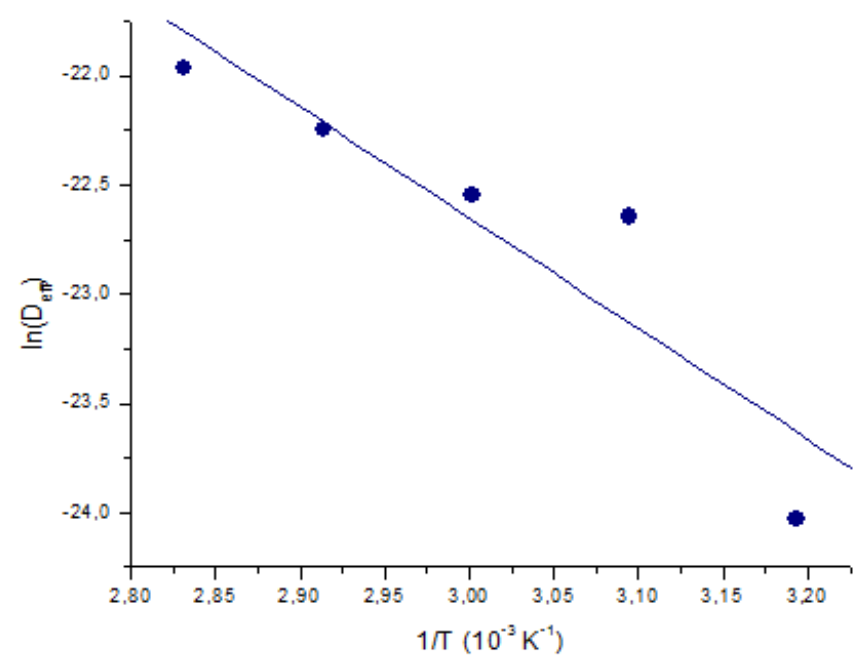

$\mathrm{O}$ valor da energia de ativação verificado foi de aproximadamente $42,1421 \mathrm{~kJ} / \mathrm{mol}$. O gráfico forneceu P-valor de 0,03208 , o qual é menor que 0,05 , indicando que a temperatura teve influência significativa no processo.

A energia de ativação é uma barreira que deve ser ultrapassada para que o processo de difusão possa ser desencadeado nas sementes e nos processos de secagem, sendo assim, quanto menor a energia de ativação, maior é a difusividade de água no material. Os valores da difusividade e da energia de ativação variam com a espécie e variedade das sementes, temperatura e a velocidade do ar de secagem, entre outros fatores (Roberts, Kidd e PadillaZakour, 2008).

O valor da energia de ativação determinada no presente trabalho foi superior ao verificado por Menezes (2014) para a mesma variedade de sementes de uva secas com velocidades do ar de 0,8,1,2 e 1,6 m/s, uma vez que a autora apresentou, respectivamente, os valores de 21,6829, 33,5354 e 40,6538 kJ/mol. Clemente et al. (2008) determinaram a energia de ativação para a secagem de sementes de uva Bobal e apresentaram um valor de 74,1 $\mathrm{kJ} / \mathrm{mol}$, maior do que o observado no presente estudo, enquanto que, Roberts, Kidd e PadillaZakour (2008) encontraram valores de energia de ativação de 40,14, 30,45 e 31,47 kJ/mol para sementes de uva Riesling, Concórdia e Cabernet Franc, respectivamente. 


\section{CONCLUSÃO}

Os valores da difusividade efetiva verificados no presente trabalho variaram de $3,66 \times 10^{-11}$ a $2,88 \times 10^{-10}$, o que está de acordo com o previsto na literatura para alimentos, entre $10^{-9}$ e $10^{-11}$. Além disso, observou-se que com o acréscimo da temperatura ocorre também o aumento da difusividade efetiva, em um comportamento praticamente linear $\left(R^{2}=0,9515\right)$.

A energia de ativação do processo de secagem de sementes de uva Bordô verificado foi de aproximadamente $42,1421 \mathrm{~kJ} / \mathrm{mol}$. O ajuste da equação de Arrhenius forneceu P-valor menor que 0,05 , indicando que a temperatura teve influência significativa no processo.

\section{REFERENCIAS}

CAMARGO, U. A.; TONIETTO, J.; HOFFMANN, A. Progressos na viticultura brasileira. Rev. Bras. Frutic, v. 33, n. 1, p. 144-149, 2011.

CHOI, Y.; LEE, J. Antioxidant and antiproliferative properties of a tocotrienolrich fraction from grape seeds. Food Chem. v. 114, n.4, p. 1386-1390, 2009.

CLEMENTE, G.; BON, J.; BENEDITO, J.; SANJUÁN, N.; MULET, A. Temperature influence on grape seeds dehydration. 16th International Drying Symposium, Hyderabad, Índia, 2008.

COSTA, J.E.; BELCHIOR, A.P. Laboração e utilização dos produtos secundários da vinificação. Relatório nacional, Lisboa, 1972.

CRANK, John. The Mathematics of Diffusion: 2d Ed. Clarendon Press, 1975.

FERNANDES, L.; CASAL, S; CRUZ, R.; PEREIRA J.A.; RAMALHOS E. Seed oils of ten traditional Portuguese grape varieties with interesting chemical and antioxidante properties. Food Res. Int.l, v. 50, p. 66-161, 2013.

GONELI, A.L.D.; CORRÊA, P.C.; AFONSO JÚNIOR, P.C.; OLIVEIRA, G.H.H. Cinética de secagem dos grãos de café descascados em camada delgada. Revi. Bras. Armaz., Viçosa-MG, n.11, p.64-73, 2009. Especial Café.

MADAMBA, P. S.; DRISCOLL, R. H.; BUCKLE, K. A. Thin layer drying characteristics ofgarlic slices. J. Food Eng., v.29, n.1, p.75-97, 1996.

McCABE, W.L.; SMITH, J. C.; HARRIOTT, P. Unit Operations of Chemical Engineering, 4th Ed., McGraw-Hill International Book Co., Singapore, 1976.

MENEZES, M. L. Estudo dos processos de secagem e de extração da produção do óleo bruto de semente de uva. 2014. 288 f. Tese (Doutorado em Engenharia Química) Universidade Estadual de Maringá, Maringá-PR, 2014.

ROBERTS, J. S.; KIDD, D. R.; PADILLA-ZAKOUR, O. Drying kinetics of grape seeds. $J$. Food Eng., v. 89, p. 460-465, 2008. 